\title{
Validation of the murine aortic arch as a model to study human vascular diseases
}

\author{
Christophe Casteleyn, ${ }^{1}$ Bram Trachet, ${ }^{2}$ Denis Van Loo, ${ }^{3}$ Daniel G. H. Devos, ${ }^{4}$ Wim Van den Broeck, ${ }^{1}$ \\ Paul Simoens ${ }^{1}$ and Pieter Cornillie ${ }^{1}$ \\ ${ }^{1}$ Department of Morphology, Faculty of Veterinary Medicine, Ghent University, Merelbeke, Belgium \\ ${ }^{2}$ Institute of Biomedical Technology, Ghent University, Ghent, Belgium \\ ${ }^{3}$ Centre for X-Ray Tomography, Ghent University, Ghent, Belgium \\ ${ }^{4}$ Department of Radiology, Ghent University Hospital, Ghent, Belgium
}

\begin{abstract}
Although the murine thoracic aorta and its main branches are widely studied to gain more insight into the pathogenesis of human vascular diseases, detailed anatomical data on the murine aorta are sparse. Moreover, comparative studies between mice and men focusing on the topography and geometry of the heart and aorta are lacking. As this hampers the validation of murine vascular models, the branching pattern of the murine thoracic aorta was examined in 30 vascular corrosion casts. On six casts the intrathoracic position of the heart was compared with that of six younger and six older men of whom contrast-enhanced computer tomography images of the thorax were three-dimensionally reconstructed. In addition, the geometry of the human thoracic aorta was compared with that of the mouse by reconstructing micro-computer tomography images of six murine casts. It was found that the right brachiocephalic trunk, left common carotid artery and left subclavian artery branched subsequently from the aortic arch in both mice and men. The geometry of the branches of the murine aortic arch was quite similar to that of men. In both species the initial segment of the aorta, comprising the ascending aorta, aortic arch and cranial/superior part of the descending aorta, was sigmoidally curved on a cranial/superior view. Although some analogy between the intrathoracic position of the murine and human heart was observed, the murine heart manifestly deviated more ventrally. The major conclusion of this study is that, in both mice and men, the ascending and descending aorta do not lie in a single vertical plane (non-planar aortic geometry). This contrasts clearly with most domestic mammals in which a planar aortic pattern is present. As the vascular branching pattern of the aortic arch is also similar in mice and men, the murine model seems valuable to study human vascular diseases.
\end{abstract}

Key words aorta; geometrical analysis; man; medical imaging; micro-computer tomography; mouse; vascular disease.

\section{Introduction}

In recent years, several researchers have investigated the hemodynamics in the murine aortic arch, which serves as a model to study human vascular diseases (Feintuch et al. 2007; Suo et al. 2007; Trachet et al. 2009). One of the most intensely studied hemodynamic factors is wall shear stress because it largely contributes to the development of atherosclerosis and aneurysms (Malek et al. 1999; Boussel et al.

Correspondence

Christophe Casteleyn, Department of Morphology, Faculty of

Veterinary Medicine, Ghent University, Salisburylaan 133, B-9820

Merelbeke, Belgium. T: +32 926477 15; F: +32 926477 90;

E: Christophe.Casteleyn@UGent.be

Accepted for publication 5 February 2010

Article published online 19 March 2010
2008). As wall shear stress cannot be measured directly, computational fluid dynamics simulations are used to quantify this phenomenon (Moore et al. 1999; Mori et al. 2003; Feintuch et al. 2007; Huo et al. 2008). These simulations are often based on vascular casts that accurately represent the in-vivo geometries and dimensions of the vascular system (Moore et al. 1999). Although both geometry and dimensions are of major importance for a full understanding of the hemodynamics (Holt et al. 1981; Feintuch et al. 2007), it has been demonstrated that variations in arterial geometry are the main cause of the variability in wall shear stress distribution (Mark et al. 1989). As a result, detailed anatomical descriptions of the murine aorta are indispensible. Such descriptions could also be useful for toxicological studies as numerous chemicals, including drugs, can produce aortic arch malformations in mice (Monnereau et al. 2005).

Notwithstanding the fact that the mouse is frequently used to model human vascular diseases, anatomical studies 
on its vascular system have seldom been performed (Szczurkowski et al. 2007). A solid knowledge of the murine circulatory system is, however, a prerequisite for the correct interpretation of experimental data and the validation of the mouse models. Furthermore, anatomical and physiological analogies between mice and humans are most controversial. For instance, Feintuch et al. (2007) state that the hemodynamic patterns in the murine aorta are substantially similar to those in humans and larger domestic mammals because of the similarity in anatomy, blood flow velocity, cardiac output waveform shape and blood pressure. In contrast, Suo et al. (2007) hold that, given the differences in size, heart rate and blood ejection fraction, it is expected that the mouse model does not scale up to the human and that the hemodynamic parameters could be quite different.

These literature data clearly demonstrate the need for detailed anatomical studies in mice. The first aim of the present study was therefore to describe the anatomy and geometry of the murine heart and thoracic aorta with its main branches. Vascular corrosion casts, which are accurate $3 \mathrm{D}$ replicas of the vascular system, were chosen for this description. Our second goal was to validate the use of the murine heart and thoracic aorta as a model to mimic human vascular diseases. For this purpose, 3D models based on contrast-enhanced computer tomography (CT) scans of six younger and six older male human hearts and thoracic aortas were compared with the 3D casts of the respective murine structures.

\section{Materials and methods}

\section{Subjects}

\section{Mice}

Thirty 2-month-old female mice (species: Mus musculus; strain: $\mathrm{C} 3 \mathrm{HeB} / \mathrm{FeJ}$ ) with a mean weight of $30.6 \mathrm{~g}$ were obtained from The Jackson Laboratory (ME, USA). Their accommodation and husbandry conformed to the guidelines stipulated in Appendix A of the European Convention for the protection of vertebrate animals used for experimental and other scientific purposes (Anonymous, 2006). Approval for this research was obtained by the Ghent University Ethical Committee on Laboratory Animal Tests (ECD 07/20).

\section{Men}

Contrast-enhanced CT images of the hearts and thoracic aortas of six younger men (aged 25-29 years) and six older men (aged 84-85 years) were analyzed in the present study. Patients, having had a CT examination of the thorax, were selected from our image archive on the basis of their age, the clinical reason for the CT investigation and the normal-for-age appearance of the aorta as assessed by a radiologist. The selected patients had their CT scans for reasons other than aortic pathology. Approval of the ethical committee of Ghent University Hospital for this retrospective study using anonymized CT scans was obtained (EC UZG 2009/648).

\section{Vascular analysis in mice}

\section{Vascular corrosion casting technique applied in mice}

The protocol of vascular corrosion casting was analogous to that described by Callewaert et al. (2008). In brief, the animals were killed by $\mathrm{CO}_{2}$ inhalation (Anonymous, 2001). A large median incision from the pubis to xiphoid was made and the abdominal aorta was catheterized with a 26 gauge catheter (Terumo Europe, Leuven, Belgium). Batson's \#17 solution (VWR, Haasrode, Belgium) ( $2 \mathrm{~mL}$ ) was gently injected free-hand in the retrograde direction. Subsequently, the mouse bodies were macerated in $25 \%$ potassium hydroxide (Roth, Karlsruhe, Germany), rinsed with water and dried under a vented hood. The casts were evaluated and photographed with a stereomicroscope (Olympus SZX7, Olympus Belgium, Aartselaar, Belgium) equipped with a charge-coupled device camera (Olympus Colorview, Olympus Belgium). All measurements on murine blood vessels were made directly on the casts under the stereomicroscope using image analysis software (Cell*D, Olympus Belgium).

\section{Intrathoracic position of the murine heart and aortic arch}

The position of and approach to the murine heart and aortic arch are described using the adjectives ventral vs. dorsal and cranial vs. caudal according to veterinary anatomical nomenclature (Anonymous, 2005).

The position of the murine heart and aortic arch into the thorax was determined on six vascular corrosion casts using image analysis software (Cell*D, Olympus Belgium). The deviation of the ventricular axis to the left of the median plane was determined on both a ventral and a cranial view. On the latter view, the deviation of the aortic arch to the left of the median plane was also measured. On a lateral view, the deviation of the ventricular axis to the ventral side of the thorax and the angle between the ventricular axis and the ascending aorta were calculated. A visual representation of the angles that were measured is given in Fig. 1A.

\section{Geometry of the murine thoracic aorta and its branches}

The geometry of the murine aortic arch and its branches was examined in six animals after carefully dissecting these structures out of the vascular casts. Three-dimensional computer models of these structures were generated by scanning the casts using an in-house-developed micro-CT system consisting of an open type X-ray system (Feinfocus, Stamford, CT, USA) with transmission target. The detector was a Very High Resolution (VHR) (Photonics Science, Millham, UK) used in binning 4, resulting in $967 \times 644$ pixels. A total of 665 projections were taken, each with $2 \mathrm{~s}$ exposure time. This was performed at $80 \mathrm{kV}$, resulting in a spot size of $3 \mu \mathrm{m}$. The images were reconstructed with OCTOPUS, which is an in-house-developed software package (Dierick et al. 2004). After cropping, 600 slices of $474 \times 407$ pixels with a pixel size of $18 \mu \mathrm{m}^{2}$ remained. The resulting images were further processed in MIMICS $^{\circledR}$ (Materialise, Leuven, Belgium) to produce a realistic 3D computer model of the aortic arch including its three side branches. An operator-defined threshold was used for the reconstruction of the scanned images into a 3D model. In MIMICS ${ }^{\circledR}$ all superfluous parts at the inlets and outlets were removed, the model surface was smoothed to remove occasional bulges and dents, and a triangular surface mesh of the 3D model was created. 


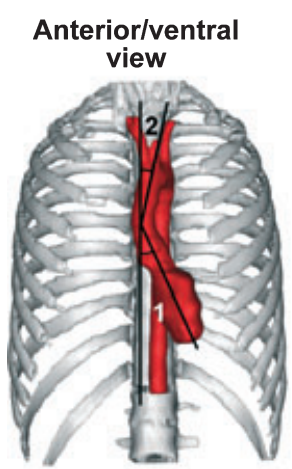

A

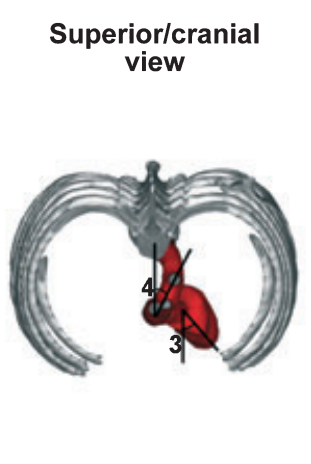

view

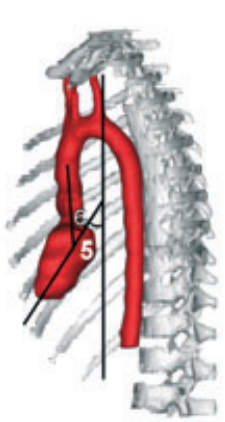

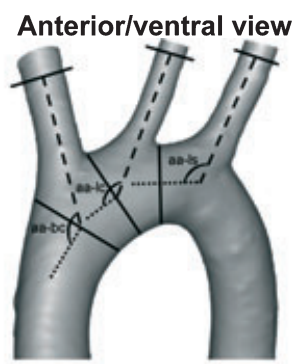

Righ lateral view

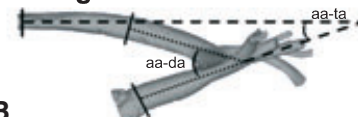

Fig. 1 Visual representations of the various angles that were measured concerning the intrathoracic position of the heart and the geometry of the aortic arch. (A) The deviations of the ventricular axis (1) and aortic arch (2) to the left of the median plane were measured on an anterior/ventral view. The same deviations (3 and 4, respectively) were also determined on a superior/cranial view. On a lateral view the deviation of the ventricular axis to ventral (5) and the angle between the ventricular axis and ascending aorta (6) were measured. (B) The angles at which the brachiocephalic trunk (aa-bc), left common carotid artery (aa-lc) and left subclavian artery (aa-ls) branch off from the aortic arch were measured on an anterior/ventral view. The angles between the ascending aorta and descending aorta at the level of the aortic bulb (aa-da) and between the ascending aorta and thoracic aorta after it had joined the vertebral column (aa-ta) were determined on a right lateral view.

In order to determine the angles at which the aortic branches originated from the aortic arch as accurately as possible, a centerline was calculated in $3-$ MATICS $^{\circledR}$ (Materialise). The bestfitting line was calculated through each branch, tangential to the centerline at that location. A plane was then defined through the endpoints of the two lines indicating the direction of the branch and the direction of the aorta directly preceding that branch. Both lines were projected onto this plane and the angle between those projections was calculated. This procedure was repeated for each of the three side branches of each model. To determine the angle between the ascending and descending or thoracic aorta, a plane was defined perpendicular to the direction of the flow, in the middle of the arch. Again, both lines indicating the direction of the ascending and descending or thoracic aorta were projected onto this plane and the angle between them was determined. As a result, only the angle of interest was taken into account and not the total (3D) angle between both lines. Figure 1B schematically shows the various angles that were measured.

\section{Vascular analysis in men}

Intrathoracic position of the human heart and aortic arch, and geometry of the thoracic aorta and its branches

The position of and approach to the human heart and aortic arch are described using the adjectives anterior vs. posterior and superior vs. inferior according to human anatomical nomenclature (Anonymous, 1998; Cook \& Anderson, 2002).

Contrast-enhanced $C T$ image datasets of six younger and six older men were generated using a SOMATOM ${ }^{\circledR}$ (Siemens AG, Muenchen, Germany) with VISIPAQUE ${ }^{\circledR}$ (lodixanol, GE Healthcare Limited, Buckinghamshire, UK) as contrasting agent. A 3D computer model was reconstructed from these $C T$ images in a similar way as mentioned above for the mice. On the 3D models the intrathoracic position of the human heart and aortic arch, and the geometry of the thoracic aorta and its branches were determined in a similar way as in mice (Fig. 1A,B). For diameter and length measurement of blood vessels a centerline was first computed for each vessel in MIMICS ${ }^{\circledR}$. Blood vessel diameters were measured orthogonally to this centerline and lengths were measured by summing the lengths of the required centerline segments. Distances between the origins of the branching vessels were measured using these centerlines.

\section{Statistical analysis}

Significant differences (when $P<0.05$ ) between the measurements performed in mice and younger and older men were detected using one-way ANOVA. All pairwise multiple comparisons were performed using the Bonferroni method.

\section{Results}

\section{Branching pattern of the aortic arch}

Mice

The brachiocephalic trunk, left common carotid artery and left subclavian artery subsequently branched off from the aortic arch in all 30 specimens (Fig. 2). The mean diameters with SE of the various segments of the murine thoracic aorta and its main branches are presented in Table 1. Table 2 shows the distances between the origins of the three branches of the aortic arch. The length of the brachiocephalic trunk ranged from 0.39 to $2.08 \mathrm{~mm}$ with a mean and SE of $1.20 \pm 0.17 \mathrm{~mm}$. In most cases the right internal thoracic artery branched off from the right subclavian artery. In four cases (13\%), however, a long brachiocephalic trunk was present, which resulted in the branching of the right internal thoracic artery from the brachiocephalic trunk (Fig. 3). 


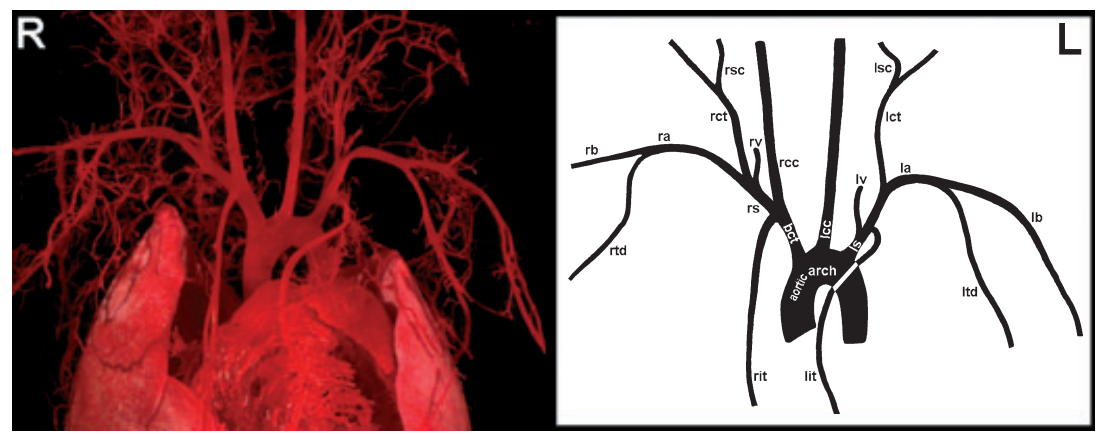

Fig. 2 Ventral view of a cast (left side of the figure) and schematically drawn (right side of the figure) murine aortic arch with its originating arteries $(\mathrm{R}$ and $\mathrm{L}=$ right and left side of the thorax, respectively). bct, brachiocephalic trunk; rit, right internal thoracic artery; rs, right subclavian artery; rcc, right common carotid artery; rv, right vertebral artery; rct, right cervical trunk; rsc, right superficial cervical artery; ra, right axillary artery; rb, right brachial artery; rtd, right thoracodorsal artery; Icc, left common carotid artery; Is, left subclavian artery; lit, left internal thoracic artery; Iv, left vertebral artery; Ict, left cervical trunk; Isc, left superficial cervical artery; la, left axillary artery; lb, left brachial artery; Itd, left thoracodorsal artery. Notice that the right internal thoracic artery in this specimen branches off from the long brachiocephalic trunk.

\section{Men}

The branching pattern of the human aortic arch was identical to that of mice (Fig. 4). The mean diameters with their SE of the various segments of the thoracic aorta and its main branches of the younger and older men are presented in Table 1. In addition, the distances between the origins of the three branches of the aortic arch are shown in Table 2. In younger men the length of the brachiocephalic trunk ranged from 36.89 to $43.59 \mathrm{~mm}$ with a mean and SE of $41.61 \pm 1.1 \mathrm{~mm}$. In older men it ranged from 11.99 to $56.50 \mathrm{~mm}$ with a mean and SE of $36.61 \pm 6.95 \mathrm{~mm}$.

\section{Anatomical topography of the heart and aortic arch}

\section{Mice}

Figure 4 shows the anatomical topography of the murine heart and aortic arch. The values of the various angles that were measured are presented in Fig. 5. The longitudinal axis of the heart was oriented obliquely in the thorax with the apex of the heart directing towards the lower left thoracic quadrant and the base of the heart together with the

Table 1 Mean diameters $(\mathrm{mm}) \pm \mathrm{SE}$ of the various segments of the thoracic aorta and its main branches of mice and younger and older men.

\begin{tabular}{|c|c|c|c|}
\hline Vessel & Mice & Younger men & Older men \\
\hline Ascending aorta & $0.82 \pm 0.01$ & $27.48 \pm 1.37$ & $35.13 \pm 2.75$ \\
\hline Aortic arch & $1.06 \pm 0.02$ & $22.74 \pm 0.97$ & $26.35 \pm 1.00$ \\
\hline Descending aorta & $0.74 \pm 0.02$ & $20.36 \pm 0.58$ & $27.96 \pm 0.72$ \\
\hline $\begin{array}{l}\text { Brachiocephalic } \\
\text { trunk }\end{array}$ & $0.54 \pm 0.02$ & $11.38 \pm 0.31$ & $16.68 \pm 1.52$ \\
\hline $\begin{array}{l}\text { Left common } \\
\text { carotid artery }\end{array}$ & $0.35 \pm 0.02$ & $7.53 \pm 0.34$ & $7.72 \pm 0.91$ \\
\hline $\begin{array}{l}\text { Left subclavian } \\
\text { artery }\end{array}$ & $0.38 \pm 0.02$ & $10.11 \pm 0.41$ & $10.91 \pm 0.83$ \\
\hline
\end{tabular}

ascending aorta directing towards the upper right thoracic quadrant. The ventricular axis deviated $31^{\circ}$ and $41^{\circ}$ to the left of the median plane on a ventral and a cranial view, respectively. On a lateral view an inclination of the ventricular axis towards the ventral side of the thorax of $39^{\circ}$ was observed.

The initial segment of the aorta, comprising the ascending aorta, aortic arch and cranial part of the descending aorta, was sigmoidally curved when looked upon from a cranial view. The ascending aorta was oriented cranially and situated ventral to the descending aorta at the right of the median plane. On a cranial view the aortic arch had an inclination of $45^{\circ}$ to the left from the median plane in the dorsocaudal direction. The descending aorta was located dorsally and to the left of the midline and pursued a caudal course. On a ventral view it was obvious that the aortic arch crossed the thorax transversely from right to left. The descending aorta was located to the left of the median plane and pursued its course in a caudal direction to join the midline at the level of the 10th thoracic vertebra.

\section{Men}

Figure 4 shows that the intrathoracic position of the human heart was quite similar to that of the murine heart. The

Table 2 Mean distances $(\mathrm{mm}) \pm$ SE between the origins of the three branches of the aortic arch in mice and younger and older men.

\begin{tabular}{llll}
\hline Branch & Mice & Younger men & Older men \\
\hline $\begin{array}{l}\text { Brachiocephalic } \\
\text { trunk - left } \\
\begin{array}{l}\text { common carotid } \\
\text { artery }\end{array}\end{array}$ & $0.96 \pm 0.06$ & $12.23 \pm 0.62$ & $16.62 \pm 3.32$ \\
$\begin{array}{l}\text { Left common } \\
\text { carotid artery - left } \\
\text { subclavian artery }\end{array}$ & $1.21 \pm 0.04$ & $21.20 \pm 2.03$ & $21.28 \pm 4.11$ \\
\hline
\end{tabular}




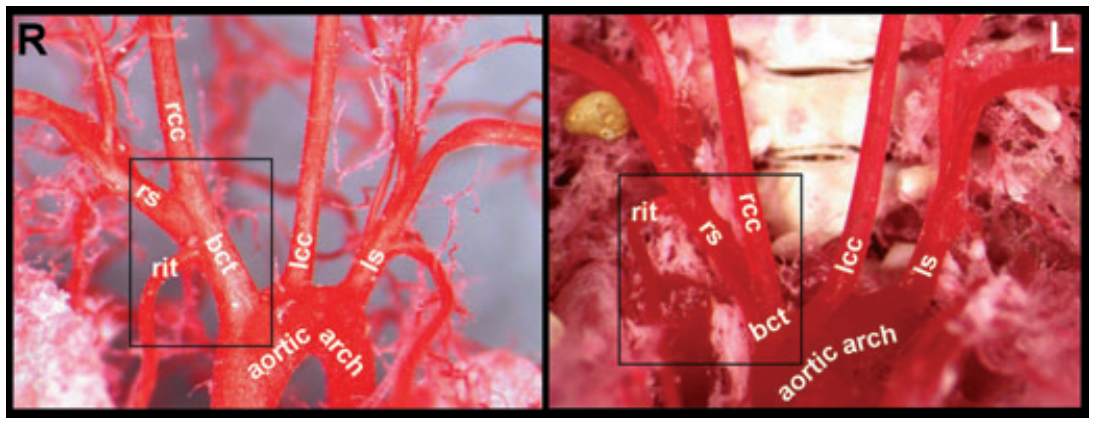

Fig. 3 Ventral views of cast murine aortic arches showing the right internal thoracic artery (rit) either branching off from a long brachiocephalic trunk (bct) (left side of the figure) or from the right subclavian artery (rs) (right side of the figure) when the brachiocephalic trunk is very short ( $R$ and $\mathrm{L}=$ right and left side of the thorax, respectively). rcc, right common carotid artery; Icc, left common carotid artery; Is, left subclavian artery.

mathematical results concerning this position are presented and statistically compared with the data of the mice in Fig. 5. Compared with mice the axis of the human heart was positioned significantly less towards the anterior side of the thorax on a lateral view (only $25^{\circ}$ and $1^{\circ}$ in younger and older men, respectively).

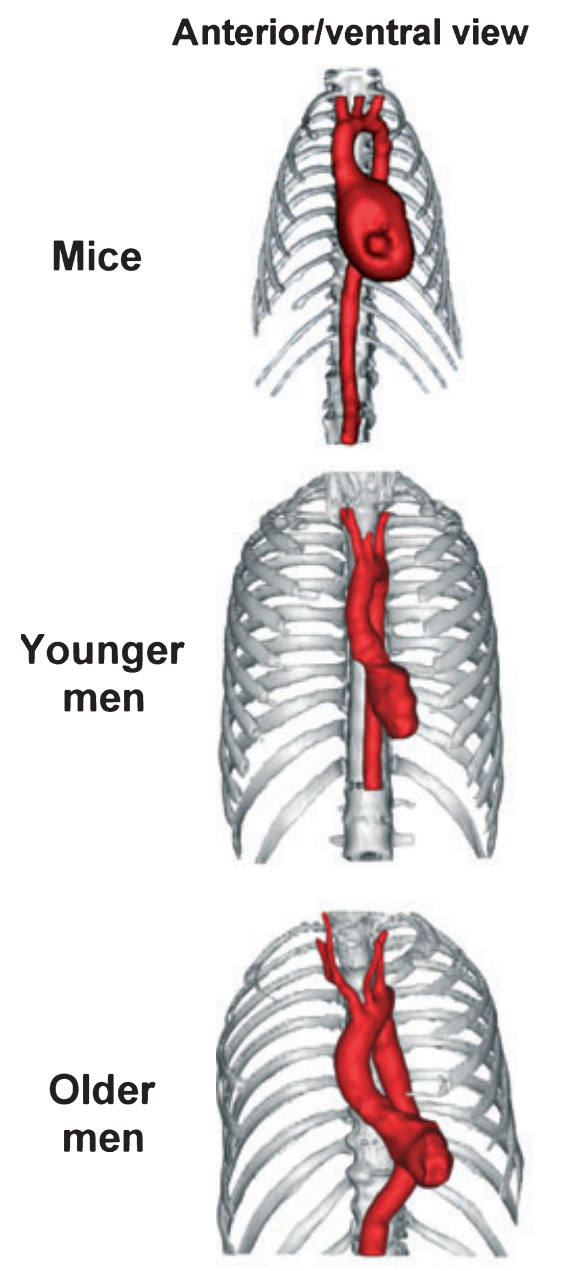

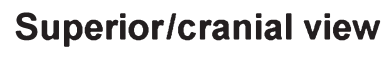
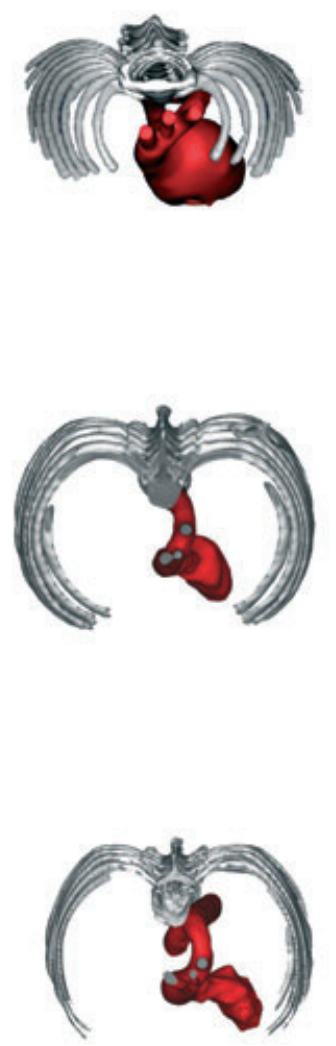

\section{Left lateral view}
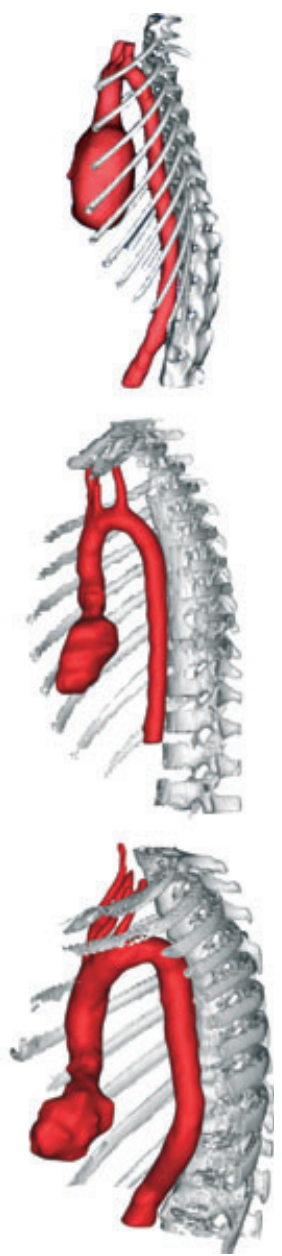

Fig. 4 Computerized 3D reconstructions of the hearts (left ventricles in men) and aortic arches of mice, younger and older men allowing the comparison of the geometry and intrathoracic position of these structures. 
A Deviation of the ventricular axis to left on anterior view

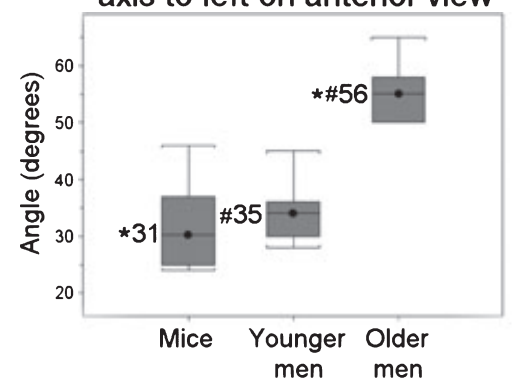

Deviation of the aortic arch

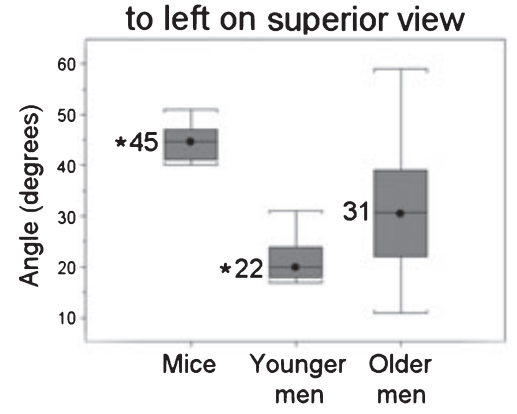

B Angle between brachiocephalic trunk and aortic arch

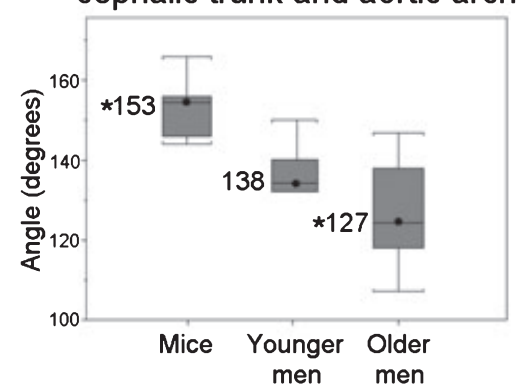

Deviation of the aortic arch

to left on anterior view

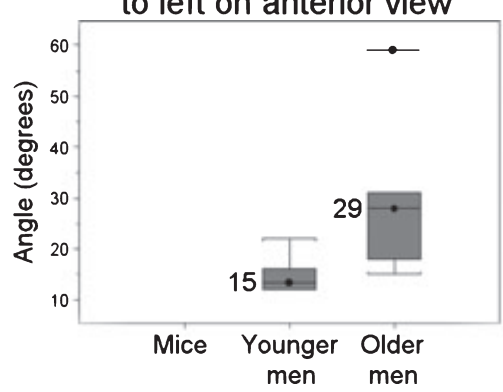

Deviation of the ventricular axis to anterior on lat. view

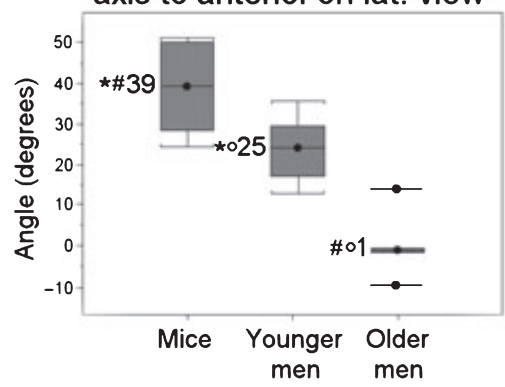

Angle between left common carotid artery and aortic arch

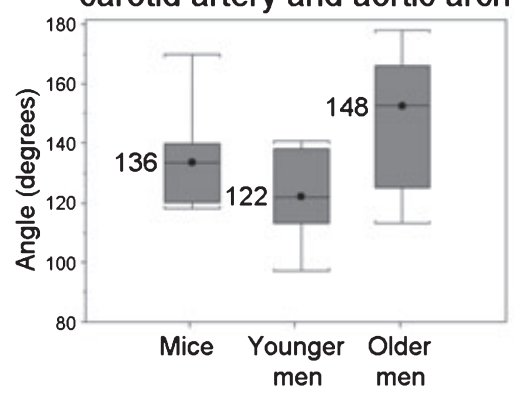

Deviation of the ventricular axis to left on superior view

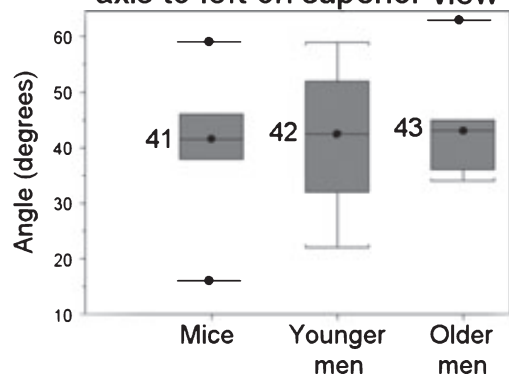

Angle between ventricular axis and asc. aorta on lat. view

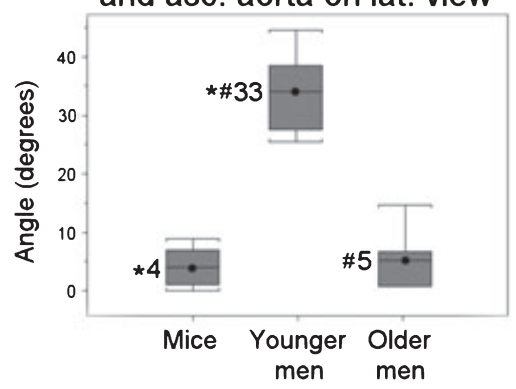

Angle between left subclavian artery and aortic arch

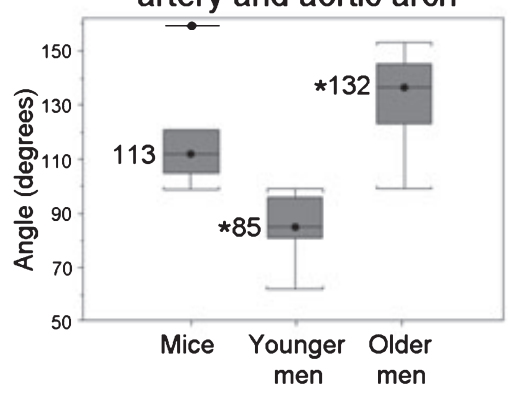

Angle between ascending

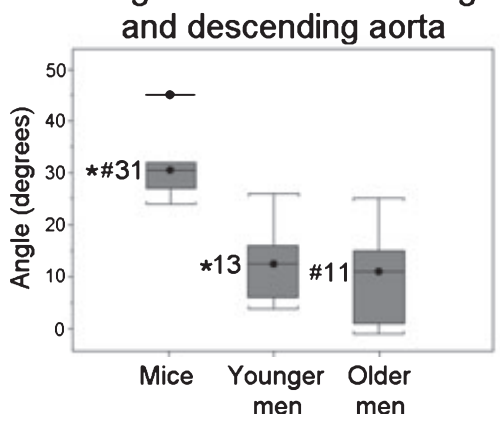

Angle between ascending and thoracic aorta

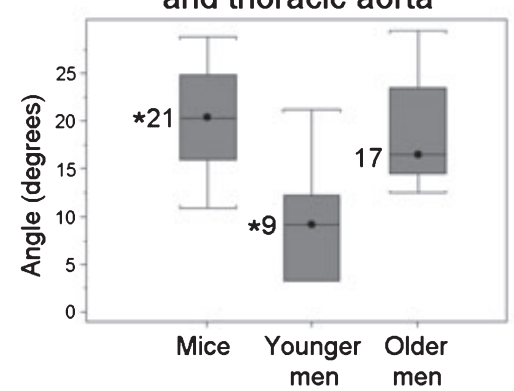

Fig. 5 Box plots showing the values of the various angles that were measured in mice, younger and older men as illustrated in Fig. $1 A, B$ ( $A$ and $B$ of the present figure, respectively). Values marked with the same symbol $\left({ }^{*},{ }^{\circ}\right.$ or \#) differ significantly from each other. The mean value is represented by the horizontal bar in the box, the box itself represents the interquartile range (25-75\% of the values), and the minimal and maximal values are marked by the horizontal bars at the lower and upper ends of the box, respectively.

As in mice, the initial segment of the human aorta was sigmoidally curved. On the anterior view the origin of the ascending aorta was positioned in the median plane (i.e. in the center of the coronal plane). The ascending aorta first ran in a superior direction, slightly to the right side of the median plane. Subsequently, it deviated to 
the left of the median plane at an angle of approximately $15^{\circ}$ in younger men and $29^{\circ}$ in older men. Such a deviation was not present in mice. On the superior view the aortic arch turned posteriorly and to the left at an angle of approximately $22^{\circ}$ and $31^{\circ}$ with the median plane in younger and older men, respectively. In younger men the descending aorta ran in a fairly straight course towards the abdomen in a prevertebral position slightly left of the median plane. In some older men the descending aorta first ran back towards the median plane and then again to the left of the median plane to join the vertebral column at the level of the 11th thoracic vertebra.

\section{Geometry of the aortic arch and its main branches}

\section{Mice}

The angles at which the brachiocephalic trunk, left common carotid artery and left subclavian artery branched off from the aortic arch are shown in Fig. 5. The angle that is present on a lateral view between the ascending and descending aorta at the level of the aortic bulb and between the ascending aorta and the thoracic aorta after the latter has joined the vertebral column is also given.

\section{Men}

Figure 5 shows the values for the younger and older men of the same five angles that were also measured in mice. Significant differences between mice, younger men and older men are indicated.

\section{Discussion}

The results of our study show that the variable length of the brachiocephalic trunk [formerly named innominate artery (Anonymous, 2005)] influences the branching pattern of the arteries that arise from it. In most examined mice the right internal thoracic artery branched off from the right subclavian artery. In a few animals, however, the right internal thoracic artery arose from a relatively long brachiocephalic trunk. These observed variations seem to be normal individual variations of the vascular system. Although an animal's vascular phenotype is mainly determined by its genotype, the environmental conditions to which the animal is exposed should not be neglected. Wilson \& Warkany (1949), for example, found that nutritional deficiency of vitamin $\mathrm{K}$ considerably increases the number of vascular varieties in the arteries that branch off from the aortic arch in the rat. Monnereau et al. (2005) found a rat fetus in which the brachiocephalic trunk was absent and the right subclavian and right common carotid arteries originated separately from the aortic arch. This finding is in accordance with the large variation in length of the brachiocephalic trunk and more specifically with the occurrence of very short brachiocephalic trunks in some mice observed in the present study.

Variation in the length of the brachiocephalic trunk is also present in humans. The mean length observed in the 12 men examined in our study was $33 \mathrm{~mm}$ with a range between 12 and $57 \mathrm{~mm}$. These values are similar to those presented by Shin et al. (2008) who found a mean length of $33 \mathrm{~mm}$ with a range between 24 and $46 \mathrm{~mm}$. In most humans the three major branches originate directly from the aortic arch (Zamir \& Sinclair, 1991). In some individuals the left common carotid artery branches off from the brachiocephalic trunk. It is also possible that the left vertebral artery originates from the aortic arch in between the left subclavian artery and left common carotid artery (Shin et al. 2008).

Figures 4 and 5 clearly demonstrate that the hearts of both mice and men are positioned obliquely in the thorax with the apex pointing to the left lower thoracic quadrant. In older men, however, the position of the heart is much more horizontal with the apex pointing to the left side of the thorax. In mice the apex of the heart manifestly points to the left ventral side of the thorax as an angle of $39^{\circ}$ is present between the ventricular axis and vertebral column. This angle is somewhat smaller in younger men and almost absent in older men. This species difference could be explained by the latero-lateral flattening of the murine thorax. A morphological explanation for the difference between younger and older men is, however, not obvious. Although the apex of the murine heart presents this ventral deviation, there is almost a straight course from the ventricle towards the ascending aorta as the latter forms an angle of $31^{\circ}$ with the descending aorta. Such an almost straight course is also seen in older men.

The ascending and descending aorta of mice are not positioned in a single vertical plane (non-planar aortic geometry). This characteristic configuration is also present in humans, albeit least pronounced in younger men. Our measurements of the inclination of the aortic arch to the left of the median plane are in accordance with the results obtained by Shin et al. (2008) who found an average inclination angle of the aortic arch of $28^{\circ}$ with variation between $0^{\circ}$ and $90^{\circ}$. Only in men was a marked inclination between the aortic arch and ascending aorta to the left side of the thorax seen on the anterior view. In mice, the transition between both aortic segments is more fluent and the aortic arch is positioned more transversely in the thorax.

In contrast to mice and younger men in whom a roman aortic arch with normal semi-circular morphology was present, the older men had gothic aortic arches with a triangular morphology (Ou et al. 2005). Such a distortion of the aortic arch is a risk factor for aneurysm development (Mori et al. 2003) and is considered as an influencing factor in the development of atherosclerosis, rather than an expression 
of the disease (Smedby \& Bergstrand, 1996). As the vascular branching pattern of the aortic arch and the geometry of the branches are also similar in mice and humans, the murine model seems valuable to study human vascular diseases. This conforms with the statement of Feintuch et al. (2007) that the anatomy of the murine and human aortic arches is quite similar. Moreover, a preliminary study performed by Casteleyn et al. (2008) indicated that the murine model appears to be far more suitable than that of other laboratory animals, such as the rabbit and rhesus monkey, or pet animals, including the cat and dog. Further research on the geometry of the aortic arches of various domestic animals should, however, be performed to definitely conclude that the mouse is the most suitable animal model to study human vascular diseases that are related with vessel geometry.

\section{Conclusion}

This study revealed that the topography of the murine and human hearts and the branching patterns of their aortic arches present many analogies. Moreover, a non-planar geometry of the aortic arch is present in both species. The aortic arches of mice and older men seem, however, to share most similarities. Therefore, the choice of many researchers to use the mouse as a model to mimic human vascular diseases, which are related to the geometrical characteristics of the thoracic aorta, seems justified.

\section{Acknowledgements}

We are grateful to $\operatorname{Dr} B$. Callewaert for providing the animals. The constructive comments made by Prof. Dr K. D'Herde and Prof. Dr M. Espeel while preparing the manuscript are kindly acknowledged. This study was partly financed by BOF grant \#05B01906, Ghent University.

\section{Author contributions}

C.C. was responsible for the design of the study, the vascular corrosion casting of the murine aortas and the drafting of the manuscript. B.T. performed the geometrical analyses of the aortas that were scanned by D.V.L. (mouse) and D.G.H.D. (human). W.V.d.B. and P.S. critically reviewed the manuscript and P.C. approved it.

\section{References}

Anonymous (1998) Terminologia Anatomica: International Anatomical Terminology. Stuttgart: Georg Thieme Verlag. Federative Committee on Anatomical Terminology (FCAT).

Anonymous (2001) Report of the AVMA Panel on euthanasia. J Am Vet Med Assoc 218, 669-696.

Anonymous (2005) Nomina Anatomica Veterinaria, 5th ed. Hamburg, Columbia, Ghent, Sapporo: World Association of Veterinary Anatomists. Internet reference: http://www. wava-amav.org
Anonymous (2006) Appendix A of the European convention for the protection of vertebrate animals used for experimental and other scientific purposes (ETS No. 123): Guidelines for accommodation and care of animals. Council of Europe, Strasbourg, 15 June 2006. Available at: http://conventions. coe.int.

Boussel L, Rayz V, McCulloch C, et al. (2008) Aneurysm growth occurs at region of low wall shear stress: patient-specific correlation of hemodynamics and growth in a longitudinal study. Stroke 39, 2997-3002.

Callewaert BL, Loeys BL, Casteleyn C, et al. (2008) Absence of arterial phenotype in mice with homozygous sIc2A10 missense substitutions. Genesis 46, 385-389.

Casteleyn C, Cornillie P, Simoens P (2008) Comparative anatomical study of the aortic arch of various animals as potential models to study human vascular disease. Poster abstract. In Proceedings of the 35th BCLAS Symposium, Charleroi, Belgium, pp. 53-54.

Cook AC, Anderson RH (2002) Attitudinally correct nomenclature. Heart 87, 503-506.

Dierick M, Masschaele B, Van Hoorebeke L (2004) Octopus, a fast and user-friendly tomographic reconstruction package developed in LabView (R). Meas Sci Technol 15, 1366-1370.

Feintuch A, Ruengsakulrach P, Lin A, et al. (2007) Hemodynamics in the mouse aortic arch as assessed by MRI, ultrasound, and numerical modeling. Am J Physiol Heart Circ Physio/ 292, H884-H892.

Holt JP, Rhode EA, Holt WW, et al. (1981) Geometric similarity of aorta, venae cavae, and certain of their branches in mammals. Am J Physio/ 241, R100-R104.

Huo YL, Guo XM, Kassab GS (2008) The flow field along the entire length of mouse aorta and primary branches. Ann Biomed Eng 36, 685-699.

Malek AM, Alper SL, Izumo S (1999) Hemodynamic shear stress and its role in atherosclerosis. JAMA 282, 2035-2042.

Mark FF, Bargeron CB, Deters OJ, et al. (1989) Variations in geometry and shear rate distribution in casts of human aortic bifurcations. J Biomech 22, 577-582.

Monnereau L, Carretero A, Berges S, et al. (2005) Morphometric study of the aortic arch and its major branches in rat fetuses on the 21st day of gestation. Anat Embryol 209, 357-369.

Moore JA, Rutt BK, Karlik SJ, et al. (1999) Computational blood flow imaging based on in vivo measurements. Ann Biomed Eng 27, 627-640.

Mori D, Tsubota K, Wada S, et al. (2003) Three dimensional configuration of the aorta determines its susceptibility to aneurysm through concentrated wall shear distribution pattern. Atherosclerosis suppl 4, 208.

Ou P, Balleux F, Jolivet O, et al. (2005) Architecture de la crosse aortique et circulation des fluides. Arch Mal Coeur Vaiss 98, 757-759.

Shin I-J, Chung Y-G, Shin W-H, et al. (2008) A morphometric study on cadaveric aortic arch and its major branches in 25 Korean adults: the perspective of endovascular surgery. $J$ Korean Neurosurg Soc 44, 78-83.

Smedby O, Bergstrand L (1996) Tortuosity and atherosclerosis in the femoral artery: what is cause and what is effect? Ann Biomed Eng 24, 474-480.

Suo J, Ferrara DE, Sorescu D, et al. (2007) Hemodynamic shear stresses in mouse aortas - Implications for atherogenesis. Arterioscler Thromb Vasc Biol 27, 346-351. 
Szczurkowski A, Kuchinka J, Nowak E, et al. (2007) Topography of arterial circle of the brain in Egyptian spiny mouse (Acomys cahirinus, Desmarest). Anat Histol Embryol 36, 147-150.

Trachet B, Swillens A, Van Loo D, et al. (2009) The influence of aortic dimensions and boundary conditions on calculated wall shear stress in the mouse aortic arch. Comput Methods Biomech Biomed Engin, 12, 491-499.
Wilson GJ, Warkany J (1949) Aortic arch and cardiac anomalies in the offspring of vitamin A deficient rats. Am J Anat 99, 113-155.

Zamir M, Sinclair P (1991) Origin of the brachiocephalic trunk, left carotid, and left subclavian arteries from the arch of the human aorta. Invest Radiol 26, 128-133. 